\title{
INVARIANT SETS FOR TRANSLATION-PARAMETER FAMILIES OF MEASURES
}

\author{
By D. BASU ${ }^{1}$ AND J. K. Ghosh ${ }^{2}$ \\ Indiana Statistical Institute
}

1. Introduction. In this paper we discuss a number of problems which have their origin in statistics but whose main interest is measure-theoretical. It is to the statistician interested in abstract harmonic analysis and to the harmonic analyst interested in statistics that the paper is addressed.

Let $P=\{P\}$ be a family of probability measures on an arbitrary measurable space $(X, Q)$. The set $A \varepsilon Q$ is called ' $P$-invariant' (in preference to the more familiar expression 'similar region') if $P(A)$ is a constant in $P$. The class $Q(P)$

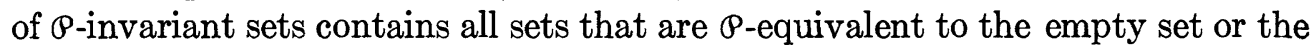
whole space, and is closed for complementation and countable disjoint unions. In general, $Q(P)$ is not a sub- $\sigma$-field of $Q$.

The set $A \varepsilon Q(\mathcal{Q})$ is 'non-trivial' if $A$ is not $\mathcal{P}$-equivalent to the empty set or the whole space. If every member of $Q(P)$ is 'trivial' then we call the family 'weakly complete'. The name is suggested by the fact that 'completeness' $\Rightarrow$ 'bounded completeness' $\Rightarrow$ 'weak completeness'. ${ }^{3}$ That weak completeness does not imply bounded completeness is seen from the example where $X$ consists of only three points with a probability distribution $\theta, \theta$ and $1-2 \theta$, where $0<\theta<\frac{1}{2}$.

If $P$ is not weakly complete, i.e., if there exist non-trivial $\rho$-invariant sets, then we call the family of measures 'weakly incomplete.' If $P$ consists of a finite number of non-atomic measures, then its weak incompleteness is an immediate consequence of a well-known result due to Liapunov [12]. In this situation the class $Q(P)$ is very wide and contains sets of all 'sizes.'

Our main concern is with the weak incompleteness of families of probability measures. Here, we restrict ourselves almost exclusively to the particular situation where $P$ is a translation parameter family of probability measures. At the risk of some repetitions, this paper brings together a number of results some of which have been noted elsewhere.

2. Notations and a few basic propositions. For the sake of simplicity of exposition we consider the case where $X$ is the additive group of real numbers.

Received 8 August 1966; revised 15 December 1967.

1 Research was done mainly at the University of Chicago and supported in part by Research Grant No. NSF GP 3707 from the Division of Mathematical, Physical and Engineering Sciences of the National Science Foundation, and in part by the Statistics Branch, Office of Naval Research. Reproduction in whole or in part is permitted for any purpose of the United States Government.

${ }^{2}$ Research was done mainly at the University of Illinois and supported partly by NSF Grant GP-3814.

${ }^{3} P$ is complete if $\int f d P=0$ for all $\mathrm{P} \varepsilon P$ implies $f=0$ a.e. $P$. $P$ is boundedly complete if $f$ is bounded and $\int f d P=0$ for all $P \varepsilon P$ implies $f=0$ a.e. $P$. 
But most of the results of this section are true for arbitrary locally compact commutative groups. We shall consider this general setting in a later section.

We take $a$ to be the $\sigma$-field of Borel sets and we denote the Lebesgue measure by $\lambda$. Though our main interest is in probability measures it is necessary for certain results to consider the class $M$ of all bounded signed measures. $M$ will be equipped with a topology in the usual way by setting norm of $\mu \varepsilon M$ as $\sup _{A}|\mu|(A)$. With this norm and convolution (denoted by $*$ ) as multiplication, $M$ becomes a Banach algebra. We denote by $M_{p}$ the class of probability measures.

For each $\mu \varepsilon M$ we have the translation parameter family $[\mu]=\left\{\mu_{\theta} \mid \begin{array}{lll}\theta & \varepsilon & X\end{array}\right\}$ where $\mu_{\theta}(A)=\mu(A-\theta)$ and $A-\theta=\{x-\theta \mid x \varepsilon A\}$. Let $Q[\mu]$ stand for the class of $[\mu]$-invariant sets, i.e., the class of sets $A$ with the property $\mu_{\theta}(A)=$ $\mu(A)$ for all $\theta$. We shall write $\bar{f}(x)$ to denote $f(-x)$.

A class of sets $B$ is translation invariant if $B \varepsilon B \Rightarrow B+t \varepsilon B$. We state below a number of results with a sketch of proof where necessary.

LEMMA 1. $Q(\mu)$ is a translation invariant monotone class which is closed with respect to complements and countable disjoint unions and contains the empty set and the whole space $X$.

LEmma 2. If $A \varepsilon$ Q and $\phi(\theta)=\mu_{\theta}(A)$ then $\lambda(A) \mu(X)=\int \phi(\theta) d \lambda(\theta)$.

Proof of Lemma 2 is an easy application of Fubini's theorem. Alternatively one may state the above lemma as: "For all unitary measures $\mu$ the convolution $\lambda * \mu=\lambda$." If $\lambda(A)=\infty$ but $\mu(X)=0$ we take $\lambda(A) \cdot \mu(X)$ as zero.

Lemma 3. If $A \varepsilon \propto[\mu]$ and $\mu(X) \neq 0$ then $\lambda(A)=0$ or infinity according as $\mu(A)=0$ or $\neq 0$.

This lemma implies that for a probability measure $\mu$ a non-trivial $[\mu]$-invariant set has infinite Lebesgue measure.

Lemma 4. If $A \varepsilon \propto[\mu]$ then $A \varepsilon \mathfrak{Q}[\mu * \nu]$ and $(\mu * \nu)(A)=\mu(A) \nu(X)$.

Corollary. If $\mu$ and $\nu$ are unitary and $A$ belongs to both $Q[\mu]$ and $Q[\nu]$ then $\mu(A)=\nu(A)$.

Proof. $\mu(A)=(\mu * \nu)(A)=(\nu * \mu)(A)=\nu(A)$ by Lemma 4.

For each measurable set $A$ let $M(A)$ be the family of measures $\mu$ such that $A$ is $[\mu]$-invariant. $M(A)$ always contains the null measure. The relation between $A$ and $M(A)$ is a dual of the relation between $\mu$ and $\propto[\mu]$. The following theorem is essentially a restatement of the preceding results.

TheOREM 1. Let $A$ be a fixed set in $a$.

(i) $M(A)$ is a closed ideal of the Banach algebra, $M$, i.e., $M(A)$ is a closed linear subspace of $M$ which is closed with respect to convolution with every $\nu \varepsilon M$.

(ii) If $M_{p}$ is the class of probability measures, then $M(A) \cap M_{p}$ is a (possibly empty) closed convex subset of $M$ and $\mu(A)$ is a constant for all $\mu$ in $M(A) \cap M_{p}$ and this common value is zero or positive according as $\lambda(A)=0$ or $\infty$.

The following two lemmas prove that we are not working in a vacuum and that weakly incomplete translation parameter families do exist.

LEMma 5. If $\mu$ be the (normalized) restriction of the Lebesgue measure $\lambda$ to the interval $J=[a, a+\delta)$ then

(i) $A \varepsilon \varepsilon[\mu]$ if and only if $A$ is an essentially periodic set with period $\delta$, i.e., 
$A \triangle(A+\delta)$ is a Lebesgue null set.

(ii) $\mathbb{Q}[\mu]$ contains sets of all sizes.

(iii) $Q$ [ $[\mu]$ is a sub- $\sigma$-field.

Proof. If $A \varepsilon Q[\mu]$ and $I_{A}$ be the indicator of $A$ then, from the fact that

$$
\int_{\theta}^{\theta+\delta} I_{A}(x) d \lambda(x) \text { is a constant in } \theta,
$$

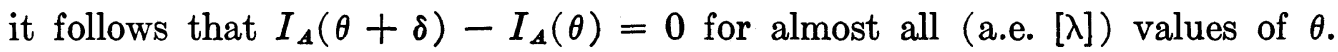
And this in turn implies the essential periodicity of $A$ with period $\delta$.

To prove the 'if' part of (i) let us first note that if $A$ be essentially periodic with period $\delta$, then, for each integer $n, A$ is essentially equal to $A+n \delta$. Also note that, for each $\theta \varepsilon X$, the sequence of sets $\{J+\theta+n \delta\}, n=0, \pm 1$, $\pm 2, \cdots$ is a partition of $X$. The [ $\mu$ ]-invariance of $A$ then follows from the following chain of equalities.

$$
\begin{aligned}
\delta \mu(A-\theta) & =\lambda[(A-\theta) \cap J]=\lambda[A \cap(J+\theta)] \\
& =\sum_{n} \lambda[A \cap(J+\theta) \cap(J+n \delta)] \\
& =\sum_{n} \lambda[(A-n \delta) \cap(J+\theta-n \delta) \cap J] \\
& =\sum_{n} \lambda[A \cap J \cap(J+\theta-n \delta)] \\
& =\lambda(A \cap J)=\delta \mu(A) .
\end{aligned}
$$

Now, if $0<\alpha<1$ and $A_{0}$ be a sub-set of $J$ such that $\lambda\left(A_{0}\right)=\alpha \delta$ and $A=$ $\mathrm{U}_{n}\left(A_{0}+n \delta\right)$ then it is clear that $A \varepsilon Q[\mu]$ and that $\mu(A)=\alpha$. This proves (ii). The proof of (iii) is elementary and hence omitted. In a later section we shall indicate how to generalize the 'if' part of (i) to general topological groups. It is easy to see how the lemma may be generalized to an arbitrary Euclidean space.

Let us contrast Lemma 5 with the following:

Lemma 6. If $\mu$ be the uniform discrete distribution over the two points $a$ and $a+\delta$ then

(i) the empty set and the whole space are the only trivial $[\mu]$-invariant sets;

(ii) $A$ is a non-trivial member of $Q[\mu]$ if and only if $A$ and $A+\delta$ are complements of each other; all such sets are of size $\frac{1}{2}$;

(iii) $\mathbb{Q}[\mu]$ is not a sub- $\sigma$-field.

A detailed proof of the above lemma is perhaps unnecessary. Only observe that $A$ is a non-trivial [ $\mu$ ]-invariant set if and only if, for every $x$ it is true that $A$ contains exactly one of the two elements $x$ and $x+\delta$. Now, for each $x$ let $S_{x}=$ $\{x+n \delta \mid n=0, \pm 1, \cdots\}$ and let $B$ be a set that has exactly one point in common with each $S_{x}$. For instance, we may take $B$ to be the interval $[a, a+\delta)$. It is now easily seen that the set $A=\mathbf{u}(B+m \delta)$ where $m$ runs through the set of even integers is a typical non-trivial member of $\alpha[\mu]$.

Lemma 6 tells us that the translation parameter family $[\mu]$ generated by any uniform two-point discrete distribution $\mu$ is weakly incomplete. It is of some interest to note that if $\mu$ is not uniform then the family $[\mu]$ is boundedly (hence 
weakly) complete but is incomplete. The generalization of Lemma 6 to the case where $\mu$ is a uniform discrete distribution on a finite number, say $n$, of points in an arithmetical progression is almost immediate. In this case we have nontrivial $[\mu]$-invariant sets of sizes $i / n(i=1,2, \cdots, n-1)$.

We thus see that weakly incomplete translation-parameter families do exist and that if $[\mu]$ is weakly incomplete then so also is $[\nu]$ if $\mu$ is a 'factor' of $\nu$ (i.e., if $\nu=\mu * \sigma)$.

In the next section we consider the case where $[\mu]$ is a dominated family of measures.

\section{The dominated case.}

3.1. Miscellaneous results.

Lemma 7. If the translation-parameter family $[\mu]$ is dominated by a $\sigma$-finite measure $\sigma$ then it is also dominated by the Lebesgue measure $\lambda$.

The proof is given by Ferguson (Lemma 2 in [8]).

In view of the above lemma in the dominated case we may take $\lambda$ as the dominating measure.

Lemma 8. If $\mu$ be a probability measure dominated by $\lambda$ then given $\epsilon>0$ there exists $\delta>0$ such that $\sup _{A}\left|\mu(A-\theta)-\mu\left(A-\theta^{\prime}\right)\right|<\epsilon$ whenever $\left|\theta-\theta^{\prime}\right|<\delta$.

The above lemma holds for an arbitrary bounded signed measure by splitting it up into its positive and negative parts. This result is well-known, Rudin ([18], p. 3). This lemma was also proved by Ferguson [7] but there is a gap in his proof; he wrongly asserts that $\lambda[A \triangle(A-\theta)] \rightarrow 0$ as $\theta \rightarrow 0$ which is true only if $\lambda(A)<\infty$. It is possible to construct an alternative proof by constructing a sequence of continuous probability densities $p_{n} \rightarrow d \mu / d \lambda$ a.e. and applying Scheffé's theorem [19].

The above lemma gives us an insight into how to construct a set that is approximately $[\mu]$-invariant. If $A$ be a periodic set with period $\delta$ then note that so also is the function $\phi(\theta)=\mu(A-\theta)$. If we choose $\delta$ sufficiently small then $A$ would be approximately $[\mu]$-invariant. The above considerations lead to the following generalization.

THEOREM 2. Let $\{f(x, \theta)\}$ be a family of probability density functions with respect to the Lebesgue measure $\lambda$ on the real line $X$ and let $\theta$ be real valued. If, for each $x \varepsilon X$, the function $f(x, \theta)$ is continuous in $\theta$, then given any $0<\alpha<1, \epsilon>0$ and $0<K<\infty$ there exists a set $A$ such that $\left|P_{\theta}(A)-\alpha\right|<\epsilon$ for $|\theta|<K$.

In the case of a dominated location-parameter family we may take $K=\infty$ and drop the assumption of continuity for $f$.

The first part of the above proposition follows from Liapunov's theorem and Scheffe's convergence theorem. It is implicitly stated in a paper of Dvoretzky, Wald and Wolfowitz [7]. The second part follows from the remarks preceding the theorem.

The following characterization of bounded completeness, vide [9], is a reformulation of a famous Tauberian theorem of Wiener.

Theorem 3. (Wiener) Let $\mu$ be dominated by the Lebesgue measure. Then [ $\mu]$ 
is boundedly complete iff the Fourier transform $\int_{-\infty}^{\infty} e^{-i t x} d \mu(x)=\hat{\mu}(t)$ does not vanish anywhere.

Wiener's theorem leads to an interesting necessary condition for weak incompleteness.

THEOREM 4. If $\mu$ is dominated by the Lebesgue measure $\lambda$ then a necessary condition that $[\mu]$ be weakly incomplete is that $\hat{\mu}(t)$ vanishes at an infinite number of points.

Proof. Let $d \mu=f d \lambda, f \varepsilon L_{1}(\lambda)$. We shall show that if $\hat{\mu}(t)=0$ only at a finite number of points, then $[\mu]$ is weakly complete. Let $\hat{\mu}(t)=0$ iff $t=t_{1}$, $\cdots, t_{m}$ and define

$$
M=\left\{\psi ; \quad \psi \varepsilon L_{\infty}(\lambda), \quad \psi(x)=\sum a_{j} e^{i t_{j} x} \text { a.e. }(\lambda)\right\} .
$$

Let $N$ be the closed ideal in $L_{1}(\lambda)$ generated by $f$. By Wiener's theorem ([18], 7.2.4), $N=\left\{\phi ; \phi \varepsilon L_{1}(\lambda), \hat{\phi}(t)=0\right.$ if $\left.t=t_{1}, \cdots, t_{m}\right\}$. Suppose $\int_{A} f(x-\theta) d \lambda=$ $c$. Then $\int_{-\infty}^{\infty}\left(I_{A}(x)-c\right) \phi(x) d \lambda=0$ if $\phi \varepsilon N$. Since $M$ is finite dimensional it follows that $I_{A}-c \varepsilon M$, i.e., $I_{A}(x)=c+\sum a_{j} e^{i t_{j} x}$ a.e. $(\lambda)$. Since every open set has positive $\lambda$-measure, the continuous function $c+\sum a_{j} e^{i t_{j} x}$ can only take the values 0 or 1 . Finally, $R$ being connected, this implies that either $I_{A}(x)=0 \quad$ a.e. $(\lambda)$ or $I_{A}(x)=1$ a.e. $(\lambda)$. This completes the proof.

Theorems 3 and 4 allow us to construct easily a $\mu$ such that $[\mu]$ is weakly complete but not boundedly complete. For example let $d \mu=x^{2}(2 \pi)^{-\frac{1}{2}} e^{-x^{2} / 2} d \lambda$. Then $\hat{\mu}(t)=\left(1-t^{2}\right) e^{-t^{2} / 2}$ which vanishes iff $t= \pm 1$.

3.2 Sufficiency and Neyman structure. We continue to consider the class of dominated measures. Equivalently we consider the class of Lebesgue integrable functions $L_{1}(X)$.

Let $\mu$ be a given dominated probablility measure with $d \mu=f d \lambda$. A natural thing to look for is a $\{\mu\}$-invariant set with Neyman structure. A set $A$ has Neyman structure if there exists a sufficient sub- $\sigma$-field $Q_{1}$ for $\{\mu\}$ and $P_{\mu}\left(A \mid Q_{1}\right)=$ constant. As the following theorem, Theorem 4 shows there exist no such nontrivial sets.

Let $Q_{s}$ be the minimal sufficient $\sigma$-field which exists by Bahadur's theorem [1].

Theorem 5. $a_{s}=Q$ wrt $\lambda$, i.e., for any $A \varepsilon$ a there exists $B \varepsilon a_{s}$ such that $\lambda(A \triangle B)=0$.

The above theorem follows from a more general result of Pitcher [17]. This theorem may be interpreted as saying the minimal sufficient sub $\sigma$-field is the maximal invariant sub $\sigma$-field under the group of transformations (in fact identity only) which leave each $P_{\theta}$ invariant. In this form the theorem is true for any separable, locally compact abelian group $G$ in place of $R$, with $m_{0}=\lambda$ taken as the Haar measure.

3.3 Characterisation of some weakly incomplete families. We confine attention to all $\mu$ dominated by $\lambda$ and identify $\mu_{f}$ with $f$ where $f \varepsilon L_{1}(\lambda)$ and $d \mu=f d \lambda$.

Two of our main problems are to characterise $M(A)$ where $M(A)$ is the class of all $\mu_{f}$ with $A \varepsilon \in \mathbb{Q}\left[\mu_{f}\right]$ (at least for some sets $A$ ) and to give conditions under which $Q[\mu]$ is a $\sigma$-field. We shall throw some light on these questions by solving a related problem. 
Consider the following problem: given a translation invariant sub- $\sigma$-field $a_{1}$ of $Q$ characterize all $f \varepsilon L_{1}(\lambda)$ such that $Q_{1} \subset Q[\mu]$ where $d \mu=f d \lambda$.

Obviously unless $Q_{1}$ is a proper sub- $\sigma$-field no such $f$ can exist.

Before this problem can be solved it is necessary to know the translation invariant proper sub- $\sigma$-fields. We do not know if the following method is the only way of generating them.

Take any countable closed subgroup $X_{1}$ of the reals i.e., $X_{1}=\{ \pm n \delta ; n=0$, $1,2, \cdots\}, \delta>0$, and consider all members $A \varepsilon Q$ which are invariant under translations by elements of $X_{1}$. Any $X_{1}$-invariant Borel set is obtained by taking a Borel set $A$ in $[0, \delta)$ and then forming the union $\mathrm{U}_{n=0, \pm 1, \ldots}(A+n \delta)$. The class of such $X_{1}$-invariant sets forms a translation invariant proper sub- $\sigma$-field of $a$, isomorphic to the class of Borel sets in $[0, \delta)$. We shall denote this class by $a_{x_{1}}$. We can prove the following:

THEOREM 6. If $a_{1}$ is a countably generated translation invariant proper sub- $\sigma$ field of $Q$, then either $Q_{1}$ is the trivial $\sigma$-field or there is a subgroup $\left\{X_{1}= \pm n \delta\right.$; $n=0,1,2, \cdots,\}>0$ such that $Q_{1}$ is the class of all $X_{1}$-invariant sets of $Q^{\text {. }}$.

Proof. Let $A$ denote an atom of $a_{1}$. Let $X_{A}=\{x ; x \varepsilon X, x+A=A\}$. Then it is easy to check that $X_{A}$ is a subgroup of $X$ not depending on $A$ and that in fact $X_{A}$ is the atom containing the origin. By [3], Theorem $3, Q_{1}$ contains all $X_{A}$-invariant measurable sets. Hence by [16], Theorem 7.2, $X_{A}$ is closed. So $X_{A}=X$ or $\{0\}$ or $\{n \delta ; n=0, \pm 1, \cdots\}, \delta>0$. The theorem follows from this.

In the following we write $a_{1}$ for $a_{X_{1}}$.

Unfortunately, though $Q$ itself is countably generated its sub- $\sigma$-fields need not have the same property. It is true that to any sub- $\sigma$-field of $a$ there corresponds countably generated sub- $\sigma$-field equivalent with respect to $\lambda$ but we are unable to prove the conjecture this suggests, namely, that if $B$ is any translation invariant sub- $\sigma$-field of $Q$ then $B=Q$ or $Q_{1}$ or the trivial $\sigma$-field wrt $\lambda$. It may be interesting to observe that if $B$ has in addition the property that there exists a set $B \varepsilon B$ with $0<\lambda(B)<\infty$, then $B=Q$ wrt $\lambda$; for define $d \mu=\left(I_{B}(x) d \lambda\right) /$ $\lambda(B)$ and apply Theorem 5 . (Alternatively this result can be directly proved and Theorem 5 derived from it.) Also see Theorem 13 in this connection.

Let us notice that the $[\mu]$-invariant sub- $\sigma$-field of Lemma 6 is equivalent to $a_{1} \operatorname{wrt} \lambda$.

In the following we write $\mu_{f}$ for $\mu$ to indicate $d \mu=f d \lambda$ but if there is no fear of confusion we shall use $\mu$ instead of $\mu_{f}$.

Theorem 7. Let $X_{1}=\{ \pm n \delta ; \delta>0, n=0,1,2, \cdots\}$ and $\mathfrak{Q}_{1}$ the sub- $\sigma$-field of all $X_{1}$-invariant sets of $Q$. Then $a_{1} \subset \mathbb{Q}\left[\mu_{f}\right]$ iff $f$ lies in the closed ideal in $L_{1}(\lambda)$ generated by $f_{\hat{\delta}}=$ characteristic ( $=$ indicator) function of the interval $[0, \delta)$.

Proof. Without loss of generality we take $\delta=1$. That $\mathfrak{Q}_{1} \subset \mathbb{Q}\left[\mu_{f}\right]$ if $f$ lies in the closed ideal generated by $f_{1}$ follows from Theorem 1 and Lemma 6 . We now prove the converse also holds. Suppose $Q_{1} \subset \mathbb{Q}\left[\mu_{f}\right]$. Then

$$
\begin{aligned}
\hat{f}(2 n \pi) & =\int_{-\infty}^{\infty} e^{-2 n \pi i x} d \mu \\
& =\int_{-\infty}^{\infty} E_{\mu}\left(e^{-2 n \pi i x} \mid Q_{1}\right) d \mu \\
& =\mu(X) \int_{-\infty}^{\infty} e^{-2 n \pi i x} f_{1} d \lambda .
\end{aligned}
$$


Since $E_{\mu}\left(e^{-2 n \pi i x} \mid Q_{1}\right)=e^{-2 n \pi i x}$ and by Lemma 5

$$
\begin{aligned}
\mu(A) \nu(X) & =\nu(A) \mu(X) \text { where } d \nu=f_{1} d \lambda, \\
& =\mu(X) \int_{0}^{1} e^{-2 n \pi i x} d \lambda \\
& =0 \text { if } n \text { is any non-zero integer. }
\end{aligned}
$$

Since $\hat{f}_{1}(t)=0$ iff $t=2 n \pi$ where $n$ is any non-zero integer, $f$ lies in the closed ideal generated by $f_{1}$ according to Theorem 7.2.4 of Rudin [18].

Theorem 8. Let $a_{1}$ be as in Theorem 7. Then

$$
\begin{aligned}
\mathfrak{a}_{1} \equiv \mathbb{Q}\left[\mu_{f}\right] \text { (up to } \lambda \text {-null sets) if } \hat{f}(t) & =0 \text { for } t=2 n \pi / \delta, \quad n= \pm 1, \pm 2, \cdots, \\
& \neq 0 \text { otherwise. }
\end{aligned}
$$

Proof. Suppose $\hat{f}(t)$ satisfies the given condition. Then $f_{\delta}$ and $f$ generate the same closed ideal, where $f_{\delta}$ is defined in Theorem 7. Hence $\mathfrak{Q}\left[\mu_{f}\right]=\mathbb{Q}\left[\mu_{f_{\delta}}\right]=a_{1}$ upto $\lambda$-null sets.

A referee has pointed out that the converse is not true, and conjectures that the theorem remains true if $\hat{f}(t)=0$ at $t=2 n \pi / \delta, n= \pm 1, \pm 2$, and at a finite number of additional points.

TheOREM 9. Let $0<\delta^{\prime}<\delta$ and $\delta^{\prime} / \delta$ be an irrational number, $A=\{x+n \delta$; $\left.0 \leqq x<\delta^{\prime}, n=0, \pm 1, \pm 2 \cdots\right\}$. Then the class $M(A)$ of all $\mu_{A}$ such that $A \varepsilon$ $a[\mu]$ is the closed ideal generated by $f_{\delta}$, where $f_{\delta}$ is the indicator function of the interval $[0, \delta)$.

Proof. It is well-known that the set of all numbers of form $m+n \xi, m, n$ integers $\xi$ irrational, is dense in $X$. Using this and Lemma 1 one can show that if $\mu \varepsilon M(A)$ then $\propto[\mu]$ contains the field generated by sets

$$
A=\left\{x+n \delta ; 0<\delta^{\prime \prime}<x<\delta^{\prime \prime \prime}<\delta, n=0, \pm 1, \pm 2, \cdots\right\},
$$

and hence being monotone $Q[\mu] \supset \mathfrak{Q}_{1}$. The result now follows from Theorem 7 .

It will be noticed that Theorem 8 gives sufficient conditions for $Q[\mu]$ to be a given sub- $\sigma$-field (of certain structure) and Theorem 9 characterizes $M(A)$ for a particular type of $A$. An $f$ satisfying the conditions of Theorem 8 is $f=\phi * f_{1}$ where $f_{1}$ is the indicator function of $[0,1)$ and $\phi$ is the standardized normal density. Both $f$ and $f_{1}$ generate the same ideal in $L_{1}(\lambda)$.

4. A more general formulation. Let us consider these problems in a somewhat more general setting. Instead of $X$ we may work with any locally compact group. But it will be sufficient for our purposes to consider a separable locally compact abelian group $G$. In particular this implies $G$ is $\sigma$-compact and hence the Haar measure, to be denoted by $m_{0}$, is $\sigma$-finite.

The method of constructing weakly incomplete families in the preceding section can be generalized as follows. Let $G_{1}$ be a countable subgroup. Suppose there exists a measurable subset $G_{0}$ of $G$ such that it has one and only one element from each coset of $G_{1}$. Note that $m_{0}\left(G_{0}\right)>0$. For $G=\bigcup_{g \varepsilon G_{1}}\left(G_{0}+g\right)$ is the countable union of disjoint sets of equal measure $m_{0}\left(G_{0}\right)$. We further assume $m_{0}\left(G_{0}\right)<\infty$ and let $f_{0}=I_{G_{0}} / m_{0}\left(G_{0}\right)$ where $I$ stands for indicator func- 
tion. Then it is easily checked that if $A$ is any $G_{1}$-invariant set then $I_{A} * \tilde{f}_{0}=$ constant. As before we can construct new weakly incomplete families starting with $f_{0}$. But in this general setting it can be shown that not all weakly incomplete families are obtainable in this way; see for example the next section on the circle group. [Also Lemma 7 shows that if the real line $X$ is given the discrete topology then there exist weakly incomplete families but none obtainable by what we have called above the method of 3.3. However in this case the group is not even $\sigma$-compact.] The known structure of locally compact abelian groups should make it quite easy to give necessary and sufficient conditions under which we can construct a weakly incomplete family as in Lemma 6 .

We conclude this section with a simple result.

THeorem 10. If a measurable $G_{0}$ exists then $G_{1}$ is closed and $[\mu]$ is constant on all $G_{1}$-invariant sets; then $\left(G, Q_{1},[\mu]\right)$ is isomorphic to $\left(G / G_{1}, B, m_{1}\right)$ where $a_{1}$ is the class of $G_{1}$-invariant sets of $Q, B$ is the class of Borel sets of the quotient $G / G_{1}$ and $m_{1}$ is the normalized Haar measure on $G / G_{1}$.

Proor. The first part follows from Theorems 5.2, 7.2 of Mackey [16]. The second part follows from Lemma 5 by taking $d \nu=f_{0} d m_{0}$ where $f_{0}$ is as in the discussion preceding this theorem.

This result shows translations on $\left(G, \mathbb{Q}_{1},[\mu]\right)$ do not provide really new examples of measure preserving transformations.

5. Circle group. It is natural that we should try to study convolutions by using Fourier transforms. The difficulty here is that if $G$ is non-compact then the indicator function of a non-trivial $[\mu]$-invariant set does not belong to $L_{1}\left(m_{0}\right)$. In order to overcome this difficulty we may work with a compact group. In this section we work with the circle group.

Let $G$ be the group of complex numbers of modulus one with multiplication as the group operation or equivalently the additive group of reals modulo $2 \pi$; we follow the second interpretation henceforth.

Since $G$ is compact the closed countable subgroups are the finite groups, in this case the cyclic groups of the form

$$
G_{1}=\left\{2 \pi K / n_{0} ; \quad K=0,1, \cdots, n_{0}-1\right\} .
$$

A typical $G_{1}$-invariant set is

$$
A=\left\{x+K 2 \pi / n_{0} ; \quad 0<x<a, \quad K=0,1, \cdots, n_{0}-1\right\}
$$

where $0<a<2 \pi / n_{0}$.

Let $G_{0}=\left\{x ; 0 \leqq x<2 \pi / n_{0}\right\}$ and $f_{0}=I_{G_{0}} /\left(2 \pi / n_{0}\right)$. As before we assume $d \mu=f(x) d m_{0}$.

In this case we can offer a different proof of Theorem 9 (and hence of Theorem 7) which is illuminating.

If $A$ is [ $\mu]$-invariant, then

$$
\begin{array}{rlll}
\hat{I}_{A}(n) \bar{f}(n) & =c & \text { if } & n=0 \\
=0 & \text { if } & & n \neq 0
\end{array}
$$


where $\hat{\phi}(n)$ for $\phi \varepsilon L_{1}\left(m_{0}\right)$ denotes the Fourier transform of $\phi$ at $n$, i.e., $\int_{0}^{2 \pi} \phi(x) e^{-i n x} d x / 2 \pi$ and $\bar{\phi}$ denotes the complex conjugate of $\phi$.

$$
\therefore \hat{f}(n)=0 \text { if } \hat{I}_{A}(n) \neq 0 \text { and } n \neq 0 \text {. }
$$

Take $A=\left\{x+2 \pi K / n_{0} ; 0<x<a, K=0,1, \cdots, n_{0}-1\right\}$ and $0<a<2 \pi / n_{0}$ with $a / \pi$ and hence $a n_{0} / 2 \pi$ an irrational number. Then

$$
\hat{I}_{A}(n) \neq 0 \quad \text { if } n= \pm K n_{0}, \quad K=1,2, \cdots .
$$

Therefore if $[\mu]$ is constant on $A$, then

$$
\hat{f}(n)=0 \quad \text { if } \quad n= \pm K n_{0}, \quad K=1,2, \cdots .
$$

Also, it is easily checked that

$$
\hat{f}_{0}(n)=0 \text { iff } n= \pm K n_{0}, \quad K=1,2, \cdots .
$$

The desired conclusion namely that $f$ lies in the closed ideal generated by $I_{A}$ follows from (1) and (2) by Theorem 7.2.4 [18].

To show that the above result about $M(A)$ is not true for all $A \varepsilon \notin$ we consider the following example suggested by the preceding arguments. Let $X=\left\{x+K 2 \pi / n_{0} ; 0<x<a, K=0,1, \cdots, n_{0}-1\right\}$ where $0<a<2 \pi / n_{0}$ and $a / \pi$ is irrational. Let $f_{1}=I_{A} / m_{0}(A)$. Then $\left[\mu_{1}\right]$ generated by $f_{1}$ is invariant on $A=\left[0,2 \pi / n_{0}\right)$. But since $\hat{f}_{1}(n) \neq 0$ if $n= \pm K n_{0}, K=1,2, \cdots$, it follows that $f_{1}$ cannot be obtained by convolution with a uniform distribution on $\left[0,2 \pi / n_{1}\right)$ for any $n_{1}$. Incidentally by Pitcher's theorem [17] the minimal suffcient sub $\sigma$-field for $\left[\mu_{1}\right]$ is properly contained in $Q$ and $A$ is not merely $\left[\mu_{1}\right]-$ invariant but also has Neyman structure.

Theorem 3 holds for any locally compact abelian group and Theorem 4 for any locally compact connected abelian group. In particular we get

THEOREM 11. If $\left[\mu_{f}\right]$ is weakly incomplete then $\hat{f}(n)=0$ at an infinite number of points.

In this case one can give an elementary proof not using Wiener's theorem.

Proof. Suppose if possible $\hat{f}(n)$ vanishes at a finite number of points. Hence if $I_{A} * \tilde{f}=$ constant, then $\hat{I}_{A}(n) \neq 0$ only at a finite number of points. Hence inversion is possible and we get

$$
I_{A}(x)=\sum e^{i n x} \hat{I}_{A}(n)
$$

which is a continuous function of $x$. Hence

$$
A=\left\{x: I_{\boldsymbol{A}}(x)=1\right\}=\left\{x: I_{\boldsymbol{A}}(x) \neq 0\right\}
$$

is both open and closed. Hence $A=G$ or $\phi$ i.e., $\left[\mu_{f}\right]$ is not weakly complete.

6. Compact groups. This section contains some remarks on other compact separable groups.

For compact groups it is easy to characterize all translation invariant sub- $\sigma$ fields. However we do not know to what extent Theorems 7, 8 and 9 hold.

Theorem 12. If $G$ is separable and compact and $a_{1}$ is a translation invariant 
sub- $\sigma$-field then there exists a compact subgroup $G_{1}$ such that $a_{1}$ differs from the class of $G_{1}$-invariant sets by $m_{0}$-null sets.

Proor. For $f \varepsilon L_{1}\left(m_{0}\right)$ let $\pi f=E_{m_{0}}\left(f \mid Q_{1}\right)$. Then it is easily seen that $\pi$ commutes with translations. Hence by Theorem 3.8.3 of [18] $\pi f=f * \mu_{0}$ where $\hat{\mu}_{0}(\gamma)=1$ or $0, \gamma \varepsilon \Gamma$ and $\Gamma$ is the dual of $G$. Let $\Gamma_{1}$ be the set of all $\gamma$ such that $\hat{\mu}_{0}(\gamma)=1$. If $\gamma \varepsilon \Gamma_{1}, \pi \gamma=\gamma * \mu_{0}=\gamma$. Hence since $\pi$ is a conditional expectation operator, $\pi(f \gamma)=\gamma \pi f$ if $\gamma \varepsilon \Gamma_{1}$. Since this holds for all $f \varepsilon L_{1}\left(m_{0}\right)$ this means if $\gamma \varepsilon \Gamma_{1}, \gamma(x)=1$ a.e. $\mu_{0}$. Since $\Gamma$ is countable and hence $\Gamma_{1}$, we can choose a $\mu_{0}$-null set $N_{0}$ such that $\gamma(x)=1$ if $x \& N_{0}$ and $\gamma \varepsilon \Gamma_{1}$. From this it easily follows that $\Gamma_{1}$ is a subgroup of $\Gamma$. Hence if $G_{1}$ is the anihilater of $\Gamma_{1}$ then the uniqueness of Fourier transforms implies $\mu_{0}$ is the normalized Haar measure corresponding to $G_{1}$. The required result now follows since $E_{m_{0}}\left(f \mid a_{1}\right)=f * \mu_{0}=E_{m_{0}}\left(f \mid a_{2}\right)$ where $a_{2}$ is the class of $G_{1}$-invariant sets of $a$.

It will be noticed that the main part of the above proof consists in giving a different and much simpler proof of Theorem 1.1 of Pitcher [17] for the compact abelian case. A proof for compact monothetic groups can be found in [1], pp. $356,357$.

If $f$ has Fourier transform that vanishes at a finite number of points, then the problem of deciding whether $\left[\mu_{f}\right]$ is weakly incomplete can be reduced to a question on finite groups. We shall not give the details since the answer is not known even for finite groups. Instead we content ourselves with stating the following which essentially shows how the reduction can be effected.

Let $\Gamma$ be the dual of $G$.

THEOREM 13. Suppose $\hat{f}$ vanishes at a finite number of points. If $[\mu]$ is weakly incomplete then

(i) the smallest subgroup $\Delta$ of $\Gamma$ containing zeros of $f$ is finite,

(ii) $G / H$ is finite where $H$ is the anihilator of $\Delta$,

(iii) there exists a set $A$ consisting of some of the cosets of $H$ such that $I_{A} * \tilde{f}=C$, $0<C<1$.

The proof is omitted. It depends on Theorem 3.3.2 of Rudin [18].

We conclude this section with two equivalent formulations of the problem of characterizing all weakly incomplete families of probability measures.

First Formulation. Which subsets $N$ of $\Gamma$ have the property $(W)$ that $N=\left\{\gamma ; I_{\Lambda}(\gamma)=0\right\}$ for some set $A$ with $0<m_{0}(A)<1$ ? [ $\mu$ ] is a weakly incomplete family of probability measure iff $N_{0}=\{\gamma ; \hat{f}(\gamma) \neq 0, \gamma \neq 0\}$, where 0 stands for the identity element of $\Gamma$, is a subset of some $N$ with property $(W)$.

Second Formulation. Given $N_{1}=\{\gamma ; \hat{f}(\gamma)=0$ or $\gamma=0\}$ where 0 is the identity element of $\Gamma$, can one construct $\phi \varepsilon L_{2}(\Gamma)$ such that $\phi=0$ outside $N_{1}$ and $\phi(\gamma)=1$ or 0 for all $\gamma$ but not equal to the indicator of $G$ or the empty set? If one can, then $A=\{x ; \hat{\phi}(x)=1\}$ is non-trivially [ $\mu$-invariant. In the case of finite groups the problem thus becomes one of characterizing the support of idempotent measures.

It seems that even for finite groups, worse still even for finite cyclic groups, the problem of characterizing all weakly incomplete families is extremely hard. 
It is interesting to note that if $G$ is a cyclic group of order $p$ where $p$ is a prime number, then the Haar measure is the unique weakly incomplete family.

7. A maximal property of the maximal invariant sub $\sigma$-field. In this section we consider an application of the concept of weak completeness to solve a problem raised by Basu [6].

Suppose $Y_{1}, \cdots, Y_{n}$ are real valued iid with density $f(x-\theta)$ (wrt Lebesgue measure), $-\infty<\theta<\infty$; let $\left(X^{(n)}, Q^{(n)}, \rho^{(n)}\right)$ denote their joint distribution and, without loss of generality, the basic space on which they are defined. Thus $d P_{\theta}{ }^{(n)}=\prod f\left(x_{i}-\theta\right) d \lambda^{(n)}$ where $\lambda^{(n)}$ is the $n$-dimensional Lebesgue measure. Clearly if $Q_{I}$ is the maximal invariant sub- $\sigma$-field induced by $Y_{2}-Y_{1}, \cdots$, $Y_{n}-Y_{1}$, then $Q_{I}$ is $\rho^{(n)}$-invariant. But even in the case of normal density with unknown mean $\theta$ and known variance it can be shown that $Q_{I}$ does not contain all $\rho^{(n)}$-invariant sets [14], p. 227. Basu [6] has raised the question whether $Q_{I}$ is a maximal $\rho^{(n)}$-invariant sub- $\sigma$-field, i.e., whether $Q_{I}$ is not contained in any $\beta^{(n)}$-invariant sub- $\sigma$-field $B$ which has at least one set $B$ such that $P_{\theta}{ }^{(n)}(A \triangle B)=P_{0}{ }^{(n)}(A \triangle B)>0$ for all $A \varepsilon Q_{I}$. Our theorem in this section gives a necessary and sufficient condition for maximality. It leads to an example showing that in general $a_{r}$ is not even maximal. Also as a corollary we have an easily verifiable sufficient condition.

Let the conditional density of $Y_{1}$ wrt $\lambda^{(1)}=\lambda$ be $f_{0}{ }^{{ }^{1}}{ }^{1}\left(x_{1} \mid Y_{i}-Y_{1}=\lambda_{i}\right.$, $i=2, \cdots, n)$. We shall write it as $f_{0}\left(x_{1}-\theta ; \lambda_{2}, \cdots, \lambda_{n}\right)$. In the following $a^{(1)}=a$ the class of linear Borel sets.

THEOREM 14. $Q_{I}$ is a maximal $\rho^{(n)}$-invariant sub- $\sigma$-field iff there does not exist any family of sets $A\left(\lambda_{2}, \cdots, \lambda_{n}\right) \varepsilon Q$ for all $(n-1)$-tuples $\left(\lambda_{2}, \cdots, \lambda_{n}\right)$ satisfying

(i) $\int_{A\left(\lambda_{2}, \cdots, \lambda_{n}\right)} f_{0}\left(x_{1}-\theta ; \lambda_{2}, \cdots, \lambda_{n}\right) d \lambda=c\left(\lambda_{2}, \cdots, \lambda_{n}\right)$

where $c\left(\lambda_{2}, \cdots, \lambda_{n}\right)$ is free of $\theta$ and $0<c<1$ on a set of $\left(\lambda_{2}, \cdots, \lambda_{n}\right)$ having positive measure under $P_{0}^{{ }^{Y_{2}}-Y_{1}, \cdots, Y_{n}-Y_{1}}$.

(ii) $A=\left\{\left(x_{1}, \cdots, x_{n}\right) ; x_{1} \varepsilon A\left(x_{2}-x_{1}, \cdots, x_{n}-x_{1}\right)\right\}$ is a $Q^{(n)}$-measurable set.

The first condition says the family of conditional densities is weakly incomplete for a set of $\left(\lambda_{2}, \cdots, \lambda_{n}\right)$ of positive probability. Condition (ii) implies these sets can be combined in a "measurable" way.

Proof. Suppose (i) and (ii) hold. Then $P_{\theta}\left(A \mid Q_{I}\right)$ is free of $\theta$; hence $P_{\theta}(B)$ is free of $\theta$ for $B \in \mathcal{F}$ where $\mathcal{F}$ is the class of finite disjoint unions of sets of form $D_{1} \cap D_{2}, D_{1}=A, A^{c}$ or $R$ and $D_{2} \varepsilon Q_{I}$. Let $B$ be the smallest $\sigma$-field containing $\mathcal{F}$. Then $\Theta$ is $P^{(n)}$-invariant. Also by (i) $A \& Q_{I}$ and so $Q$ is a proper sub- $\sigma$-field of $B$.

Conversely, suppose $a_{I}$ is contained in $a \odot^{(n)}$-invariant sub- $\sigma$-field $B$ with a set $B \varepsilon B$ such that $P_{\theta}{ }^{(n)}(A \triangle B)=P_{0}{ }^{(n)}(A \Delta B)>0$ for all $A \varepsilon Q_{I}$. Then

$$
P_{0}^{(n)}\left(A \mid Q_{I}\right)=C\left(Y_{2}-Y_{1}, \cdots, Y_{n}-Y_{1}\right)
$$

is strictly between 0 and 1 with positive probability under $P_{0}{ }^{(n)}$. Then for 


$$
\int_{A\left(\lambda_{2}, \cdots, \lambda_{n}\right)} f_{0}\left(x_{1}-\theta ; \lambda_{2}, \cdots, \lambda_{n}\right) d \lambda=C\left(\lambda_{2}, \cdots, \lambda_{n}\right)
$$

a.e. $P_{0}{ }^{Y_{2}-Y_{1}, \cdots, Y_{n}-Y_{1}}$ for $B$ being $\rho^{(n)}$-invariant we may take $P_{\theta}^{(n)}\left(A \mid Q_{I}\right)=$ $P_{0}{ }^{(n)}\left(A \mid Q_{I}\right)$. Hence there exists a set $N$ of measure zero under $P_{0}{ }^{Y_{2}-Y_{1}, \cdots, Y_{n}-Y_{1}}$ such that if $\left(\lambda_{2}, \cdots, \lambda_{n}\right) \varepsilon^{\prime} N$ then (2) holds for all rational $\theta$; by Lemma 9 . (2) holds for all $\theta$ if $\left(\lambda_{2}, \cdots, \lambda_{n}\right) \& N$. This completes the proof.

Corollary. $Q_{I}$ is a maximal $\rho^{(n)}$-invariant sub- $\sigma$-field if

$$
\int_{-\infty}^{\infty} e^{-i t x_{1}} f_{0}\left(x_{1} ; \lambda_{2}, \cdots, \lambda_{n}\right) d \lambda \neq 0
$$

for any $t$ for almost all $\left(\lambda_{2}, \cdots, \lambda_{n}\right)$ under $P_{0}{ }^{{ }^{Y_{2}}-\mathrm{Y}_{1}, \cdots, \mathrm{Y}_{n}-\mathrm{Y}_{1}}$.

The sufficient condition for maximality given in the corollary holds for the case of normally distributed random variables with mean $\theta$ and variance unity.

To show that $a_{I}$ is not always maximal consider the following example: Let $f_{0}(x)=1$ if $0 \leqq x \leqq 1$. Then

$$
f_{0}\left(x_{1} ; \lambda_{2}, \cdots, \lambda_{n}\right)=1 /\left(a_{2}-a_{1}\right) \text { if } a_{1}<x_{1}<a_{2}
$$

where $a_{1}, a_{2}$ are functions of $\left(\lambda_{2}, \cdots, \lambda_{n}\right)$ and $a_{1}<a_{2}$ with probability one under $P_{0}{ }^{Y_{2}-Y_{1}, \cdots, Y_{n}-Y_{1}}$. Let the sample space of $Y_{1}$ be divided up into left closed right open intervals $[i \lambda,(i+1) \lambda)$ where $2 \lambda=a_{2}-a_{1}, i=0, \pm 1, \pm 2, \cdots$. Let $A\left(\lambda_{2}, \cdots, \lambda_{n}\right)$ be the union of those intervals that have an even $i$ in their left end point. Then $A\left(\lambda_{2}, \cdots, \lambda_{n}\right)$ satisfies (i) and (ii) of Theorem 15 with $C\left(\lambda_{2}, \cdots, \lambda_{n}\right)=\frac{1}{2}$ for almost all $\left(\lambda_{2}, \cdots, \lambda_{n}\right)$ under $P_{0}{ }^{X_{2}-Y_{1}, \cdots, Y_{n}-Y_{1}}$.

For this example we can construct the unique maximal $\rho^{(n)}$-invariant sub- $\sigma$ field containing $Q_{I}$. Consider the class of all $A \varepsilon Q^{(n)}$ such that $P_{\theta}\left(A \mid Q_{I}\right)$ is free of $\theta$ and call it $B$. It follows from Lemma 5 and the proof of Theorem 15 that $\Theta$ is the unique maximal $\beta^{(n)}$-invariant $\sigma$-field containing $Q_{1}$. We conjecture that $B$ is in fact the class of all $\rho^{(n)}$-invariant sets.

The conjecture suggests the following general question. When is the class of all $\rho^{(n)}$-invariant sets a $\sigma$-field? For $Y_{1}, \cdots, Y_{n}$ normally distributed with mean $-\infty<\theta<\infty$ and unit variance, our corollary shows the answer is no. But as we have seen even for $n=1$ the general problem is hard to solve.

8. Problems and speculations. We have already mentioned a few problems above. We list some more below. We confine ourselves to the real line unless otherwise stated. Also we assume $[\mu]$ is dominated.

The main problem is to produce at least one weakly incomplete dominated family of probability measures $\left[\mu_{f}\right]$ which is not of the kind considered in 3.3. If no such example exists most of the questions asked below would have a trivial answer.

Let $E$ be a set with finite positive Lebesgue measure and $\mu$ the normalized restriction of Lebesgue measure to $E$. When is it true that $[\mu]$ is boundedly (weakly) complete? If $E$ is an interval (parallelogram if we are on the plane) then we know from Lemma 5 that $[\mu]$ is weakly incomplete. What is the state of affairs if $E$ is a circle or a triangle on the plane?

What are the translation invariant sub- $\sigma$-fields not covered by Theorem 6 ? 
What are the analogues of Theorems 7 and 8 for such sub- $\sigma$-fields? If $[\mu]$ has nontrivial invariant sets of all sizes then does $\propto[\mu]$ include a translation invariant sub- $\sigma$-field? Does it have a factor which is a uniform distribution over some interval?

What is the class of all sets $A$ with non-empty $M(A) \cap M_{p}$ ? What are the extreme points of $M(A) \cap M_{p}$ for such an $A$ ?

Acknowledgment. We would like to thank Professor M. Rajagopalan for some helpful discussions, and Mr. B. V. Rao who showed us how the proof of Theorem 6 can be simplified.

\section{REFERENCES}

[1] AdLer, R. A. (1964). Invariant and reducing subalgebras of measure preserving transformations. Trans. Amer. Math. Soc. 110 350-360.

[2] Bahadur, R. R. (1954). Sufficiency and statistical decision functions. Ann. Math. Statist. 25 423-462.

[3] Blackwell, D. (1956). On a class of probability spaces. Proc. Third Berkeley Symp. Math. Statist. Prob. 2 1-6. Univ. of California Press.

[4] BAsu, D. (1959). The family of ancillary statistics. Sankhyā 21 247-256.

[5] BASU, D. (1964). Recovery of ancillary information. Sankhyā 26 3-16.

[6] BAsU, D. (1965). Problems related to the existence of maximal and minimal elements in some families of statistics (sub-fields). Proc. Fifth Berkeley Symp. Math. Statist. Prob. 1 41-50. Univ. of California Press.

[7] Dvoretzky, A., WALD, A. and Wolfowitz, J. (1951). Eliminations of randomization in certain statistical decision procedures and zero sum two person games. Ann. Math. Statist. 22 1-21.

[8] Ferguson, T. S. (1963). Location and scale parameters in exponential families of distributions. Ann. Math. Statist. 33 986-1001.

[9] Ghosh, J. K. and Singh, R. (1966). Unbiased estimation of location and scale parameters. Ann. Math. Statist. 37 1671-1675.

[10] Halmos, P. R. (1948). The range of vector-measure. Bull. Amer. Math. Soc. $54416-$ 421.

[11] Halmos, P. R. (1950). Measure Theory. Van Nostrand, Princeton.

[12] Kagan, A. M. and Linnik, YU. V. (1964). A class of families of distributions with similar regions (in Russian). Vestnik Lenningrad Univ., Ser. Mat. Meh. Astronom. 19 16-18.

[13] Lehmann, E. L. and Scheffe, Henry (1950). Completeness similar regions and unbiased estimation-Part I. Sankhyā 10 305-340.

[14] Lehmand, E. L. (1959). Testing Statistical Hypotheses. Wiley, New York.

[15] Liapunov, A. (1940). Sur les functions-vecteurs completement additives. Izvestiya Akad Nauk, SSSr. Ser. Mat. 4 465-478.

[16] Mackey, G. M. (1957). Borel structures in groups and their duals. Trans. Amer. Math. Soc. 85 134-165.

[17] Pitcher, T. S. (1957). Positivity in H. Systems and sufficient statistics. Trans. Amer. Math. Soc. 85 166-175.

[18] Rudin, H. (1962). Fourier Analysis on Groups. Interscience, New York.

[19] SchefFE, H. (1947). A useful convergence theorem for probability distributions. Ann. Math. Statist. 18 434-438. 